\title{
Prevalence of anemia in adolescent girls and its co-relation with demographic factors
}

\begin{abstract}
Aims Objectives: Aim to find out prevalence of anaemia in adolescent girls and its correlation with demographic factors. Materials and Methods: 1000 adolescent girls were included in study between 12-15 years of age modified kuppuswamy scale were used to classify in socio economic groups. The nutritional status of all girls were analysed. Blood was collected for following indices Hb RDW PCV RBC count. Results: Aamong 1000 adolescent girls 60percent found to be anaemic, 18.4 percent as mild 41.3percent as moderate, and 0.4 percent was having severe anaemia. High prevalence of anaemia was reported among adolescent girls belonging to class III, IV, V as per modified kuppuswamy classification. ${ }^{[1]}$ Thin and severely thin adolescent girls were found to be at higher risk of anaemia.
\end{abstract}

Key words: Adolescent girls, anemia, socio-economic status

Neelam S. Deshpande, Devkinandan Karva, Sharad Agarkhedkar, Shishir Deshpande

Department of Pediatrics, D. Y. Patil Medical College and Hospital, Pimpri, Pune India.

Address for the Correspondence:

Dr. N. S. Deshpande, H-102, Nav Pinnac Kanchanganga

Co-Op Hsg Society,

Opp. Convergeys, D.P. Road, Aundh, Pune - 411 007,

Maharashtra, India.

E-mail: drneelam15@rediffmail.com

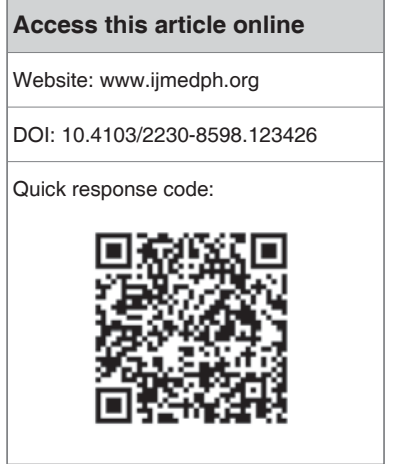

\section{INTRODUCTION}

Nutritional anemia is a global problem of immense health significance affecting persons of all age and economic group.

Anemia is one of the most common hematological abnormalities found in children. It can be defined as the reduction in oxygen-carrying capacity or as a reduction in the red cell mass of the body.

Among the various types of nutritional anemias, iron-deficiency anemia is the most common, affecting more than two billion people globally. ${ }^{[2]}$

WHO criteria for the diagnosis of anemia. ${ }^{[3]}$

Hemoglobin thresholds used to define anemia

Age or gender group

Children (0.50-4.99 yrs)

Children (5.00-11.99 yrs)

Children (12.00-14.99 yrs)

Non-pregnant woman $(\geq 15.00 \mathrm{yrs})$

pregnant woman

Men $(\geq 15.00$ yrs $)$

National Family Health Survey (NFHS) estimates reveals presence of nutritional anemia to be $70-80 \%$ in children, $70 \%$ in pregnant women, and $24 \%$ in adult women. ${ }^{[4]}$

With $40 \%$ prevalence of nutritional anemia in the world on an average for the general population, the prevalence in the developing countries tends to be three to four times higher than in the developed countries. ${ }^{\left[{ }^{[}\right.}$

In studies conducted in developing countries, adolescent anemia was reported as the greatest nutritional problem. ${ }^{[6]}$

Adolescence is a crucial phase of growth in the life cycle of an individual. It is a period of transition between childhood and adulthood occurring between 12-18 yrs of age. (Chilman and Nancy, 1994). 
Adolescents of both the sexes are particularly vulnerable to developing anemia because of rapid growth, weight gain, and blood volume expansion and in girls additionally because of onset of menstruation. In girls, middle adolescence growth happens earlier (i.e., during 12-15 yrs) than in boys (i.e., during 13-16 yrs).

Adolescent girls form a crucial segment of the population and constitute, as it were, the vital "bridge" between the present generation and the next (Raman, 1992).

In many developing countries, one half all children and adolescents fail to achieve their full genetic growth potential due to the combined effects of inadequate nutrition and frequent illness. Moreover, due to faulty dietary habits, ignorance, and in a country like India, with a multitude of social customs and beliefs cited against women, the prevalence of malnutrition amongst girls remains quite high.

Anemia not only affects the present health status, but also has deleterious effects in the future. Learning, cognitive function, and scholastic performance is also severely affected. The rates of low birth weight, pre-maturity, neonatal and infant mortality among children born to undernourished adolescent girls is high. Later on these undernourished girls becomes anemic and produce low birth weight babies.

\section{AIMS AND OBJECTIVE}

- To screen adolescent girls between 12-15 yrs of age for the prevalence of anemia

- To study its co-relation with the socio-economic status of the family

- To study the type of anemia.

\section{MATERIALS AND METHODS}

The study involved 1000 healthy girls between 12 to 15 yrs of age, out of which 500 girls were from Marathi medium school, and the remaining 500 were from English medium school. The sample size was calculated by using 'Epi Info 7,' statistical package of WHO. Taking a prevalence of $65 \%$ with confidence limit of $3 \%$ at $95 \%$ confidence level, sample size was determined to be 970 . For the sake of convenience, 1000 subjects were screened.

The study was carried out after approval by Institutional Ethical Committee and informed consent of parents/guardians.

A pre-tested and pre-designed proforma was used to collect the information on socio-demographic characteristics like age, educational status, family size, monthly family income, relevant medical history like weakness, fatigue history of worm infestation, and dietary history.

Healthy subjects not suffering from any disease were selected. Modified Kuppuswami socio-economic status was used to divide the study groups into five classes. Modified Kuppuswami socio-economic status is an important tool in hospital- and community-based research in India. This scale takes account of education, occupation, and income of family to classify study group in five classes depending upon score. Taking inflation and price rise, this has been modified with conversion factor for the income.

For classification of anemia, reference range of hemoglobin was used as per WHO classification:

- $\quad$ Mild 10-11.9 g/dL

- Moderate 7-9.9 g/dL

- $\quad$ Severe $<7 \mathrm{~g} / \mathrm{dL}$.

In all the girls, following observations were recorded - height, weight, skinfold thickness (SFT), neck circumference (NC) (1), waist-hip ratio $(\mathrm{W} / \mathrm{H})^{(2)}$, and Body Mass Index $(\mathrm{BMI})$ was calculated. The nutritional status of all girls was analyzed using WHO BMI charts for girls. ${ }^{(3)}$ Blood was collected under all aseptic precautions, and following indices were calculated- $\mathrm{Hb}, \mathrm{RDW}, \mathrm{PCV}, \mathrm{RBC}$ count, $\mathrm{MCV}$, $\mathrm{MCHC}$, and PBS was examined. All observations were recorded, tabulated, and subjected to statistical analysis. All the statistical analysis was carried out by Chi-square test and $Z$ test, in which $P<0.05$ was significant. The $P$ value was determined by using the primer of biostatistics of the SPSS software volume 11 .

\section{RESULTS}

In the present study of 1000 adolescent girls, $198(19.8 \%)$ belonged to 12 years, $201(20.1 \%)$ belonged to 13 years, 290 (29\%) belonged to 14 years, and $311(31.1 \%)$ belonged to 15 years of age.

As per WHO criteria for diagnosis and classification of anemia [Table 1], $399(39.9 \%)$ of the subjects were normal (Hb more than or equal to 12) [Figure 1], 184 (18.4\%) subjects had mild anemia (Hb between 10 and 11.9), 413 (41.3\%) subjects had moderate anemia (Hb between 7 and 9.9), and 4 subjects had severe anemia (Hb less than 7) [Table 2 and Figure 2].

Among the subjects with $\mathrm{Hb}$ less than $12 \mathrm{gm} / \mathrm{dl}$ (60\%). 70\% were observed to have low MCV with high RDW, 24.5\% had low MCV with normal RDW, and 5.5\% had high MCV with high RDW.

As per Modified Kuppuswami Scale for socio-economic status, the study group was divided into five classes. In socio-economic class I (upper) out of 224 subjects, 97 (43.3\%) subjects were reported to be anemic with 34,62, and 1 being suffering from mild, moderate, and severe anemia, respectively. In socio-economic class II (upper middle) out of 399 subjects, 195 (48.8\%) were reported to be anemic with 68,126 , and 1 being suffering from mild, moderate, and severe anemia, respectively [Table 3 and Figure 3].

In socio-economic class III (lower middle), out of 251 subjects, $204(81.2 \%)$ were reported to be anemic with 64, 140, 0 being suffering from mild, moderate, and severe anemia, respectively [Table 3].

In socio-economic class IV (upper lower) and class V (lower), out of 126 subjects, $105(83.3 \%)$ were reported to be anemic with 18,85 , and 2 suffering from mild, moderate, and severe anemia, respectively [Table 3]. 


\begin{tabular}{lcc}
$\begin{array}{l}\text { Table 1: Age-wise distribution of cases in study } \\
\text { group }\end{array}$ & \\
\hline Age (Yrs) & No of cases & Percentage \\
\hline 12 & 198 & 19.8 \\
13 & 201 & 20.1 \\
14 & 290 & 29 \\
15 & 311 & 31.1 \\
Total & 1000 & 100 \\
\hline
\end{tabular}

\begin{tabular}{lcc}
$\begin{array}{l}\text { Table 2: Hemoglobin-wise distribution of cases in } \\
\text { study group }\end{array}$ & No of cases & Percentage \\
\hline Hb (gm \%) & 399 & 39.9 \\
\hline Normal $(\geq 12)$ & 184 & 18.4 \\
Mild (10-11.9) & 413 & 41.3 \\
Moderate $(7-9.9)$ & 4 & 0.4 \\
Severe $(<7)$ & 1000 & 100 \\
Total & & \\
\hline
\end{tabular}

Table 3: Association between socio economic class and hemoglobin in study group

\begin{tabular}{lccccc}
\hline SE class & \multicolumn{4}{c}{ Hemoglobin (gm \%) } & Total \\
\cline { 2 - 5 } & Mild & Moderate & Severe & Normal & \\
\hline I & 34 & 62 & 1 & 127 & 224 \\
II & 68 & 126 & 1 & 204 & 399 \\
III & 64 & 140 & 0 & 47 & 251 \\
IV & 16 & 57 & 2 & 21 & 96 \\
V & 2 & 28 & 0 & 0 & 30 \\
Total & 184 & 413 & 4 & 399 & 1000 \\
\hline$\chi^{2}=154.73$, & $P<0.0001$ & & & &
\end{tabular}

As per WHO BMI charts 2007 for girls between 5-19 years, nutritional status was determined, and 74 subjects were reported to be normal [Table 4].

Out of remaining 276 subjects, 57, 187, 30, and 2 subjects were found to be obese, overweight, thin, and severely thin, respectively [Table 4].

Among 724 subjects with normal BMI, 488 (67.4\%) were found to be anemic with 148, 338, and 2 being suffering from mild, moderate, and severe anemia, respectively [Table 4 and Figure 4].

As per the skin fold thickness measured at the site of forcep, 951 subjects had SFT $\leq 14.5 \mathrm{~mm}$ (below normal), out of which $598(62.8 \%)$ were anemic and 353 were normal. Forty-nine subjects had SFT $>14.5 \mathrm{~mm}$, of which $3(6.1 \%)$ were anemic and 46 were normal [Table 5 and Figure 5].

A total of 817 subjects out of 1000 had neck circumference $<27 \mathrm{~cm}$, which is suggestive of undernutrition, $520(63.6 \%)$ were found to be anemic, and the rest 297 were normal. One hundred and eighty-three subjects had neck circumference within the normal range, out of which $81(42.4 \%)$ were anemic, and rest 102 subjects were normal [Table 6 and Figure 6].

Table 4: Association between body mass index
and hemoglobin in study group
\begin{tabular}{lcccccc}
\hline BMI & Mild & Moderate & Severe & Normal & \\
\hline Normal & 148 & 338 & 2 & 236 & 724 \\
Obese & 4 & 12 & 0 & 41 & 57 \\
Over weight & 27 & 42 & 0 & 118 & 187 \\
Thin & 4 & 20 & 2 & 4 & 30 \\
Severe thin & 1 & 1 & 0 & 0 & 2 \\
Total & 184 & 413 & 4 & 399 & 1000 \\
\hline
\end{tabular}

$\chi^{2}=126.66, P<0.0001, \mathrm{BMI}=$ Body Mass Index

Table 5: Association between skin fold thickness
and hemoglobin in study group
\begin{tabular}{lccccc}
\multicolumn{5}{c}{ Hemoglobin (gm \%) } & Total \\
\cline { 2 - 5 } SFT & Mild & Moderate & Severe & Normal & \\
\hline$\leq 14.5$ & 183 & 411 & 4 & 353 & 951 \\
$>14.5$ & 1 & 2 & 0 & 46 & 49 \\
Total & 184 & 413 & 4 & 399 & 1000 \\
\hline
\end{tabular}

$\chi^{2}=62.06, P<0.0001, \mathrm{SFT}=$ Skinfold thickness

\begin{tabular}{|c|c|c|c|c|c|}
\hline \multirow[t]{2}{*}{ NC } & \multicolumn{4}{|c|}{ Hemoglobin (gm \%) } & \multirow[t]{2}{*}{ Total } \\
\hline & Mild & Moderate & Severe & Normal & \\
\hline$<27$ & 161 & 355 & 4 & 297 & 817 \\
\hline $27-34.5$ & 23 & 58 & 0 & 102 & 183 \\
\hline Total & 184 & 413 & 4 & 399 & 1000 \\
\hline
\end{tabular}

\section{DISCUSSION}

In the present study, prevalence of anemia was reported to be $60 \%$. Such a high incidence is in consistent with studies conducted by Kaur et al. ${ }^{[7]}$ who reported $59.8 \%$ in adolescent girls of rural Wardha. Rana et al. ${ }^{[8]}$ and Sheshadri et al. ${ }^{[9]}$ reported similar prevalence of anemia as $60 \%$ and $63 \%$, respectively.

As per WHO classification, majority of subjects (41.3\%) suffered from moderate anemia. While $18.4 \%$ and $0.4 \%$ suffered from mild and severe anemia, respectively. Similar results were reported by CMS Rawat $e t$ al. ${ }^{[10]}$ and Warma et al..$^{[1]}$ where they noted high incidence of mild to moderate anemia.

Socio-economic and demographic factors has a bearing on prevalence of anemia. In the present study, a very high percentage of adolescent girls from low socio-economic background were found to suffer from anemia. These findings are similar to the study conducted by R. Gawarika et al. ${ }^{[12]}$ where the prevalence was found to be $96.5 \%$ in weaker income group and $65.18 \%$ in middle or higher middle group. Kapoor et al. ${ }^{[13]}$ also reported $56 \%$ in lower middle and $46 \%$ in high socio-economic group. In the present study, all 30 girls from lower socio-economic strata were observed to be anemic with 2 and 28 being suffering from mild and moderate anemia, respectively. 


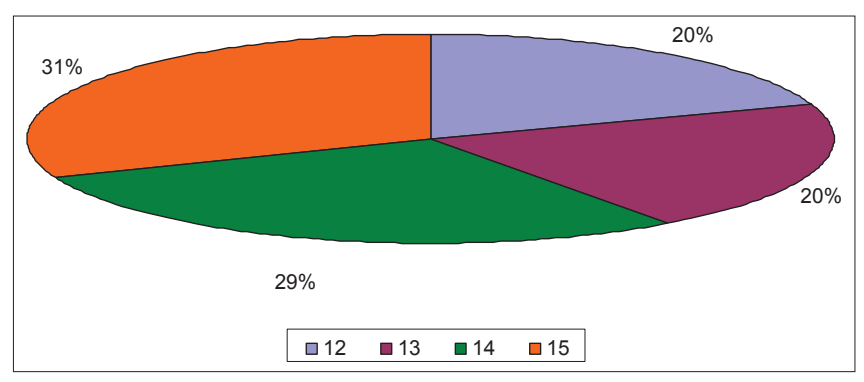

Figure 1: Age wise distribution of cases

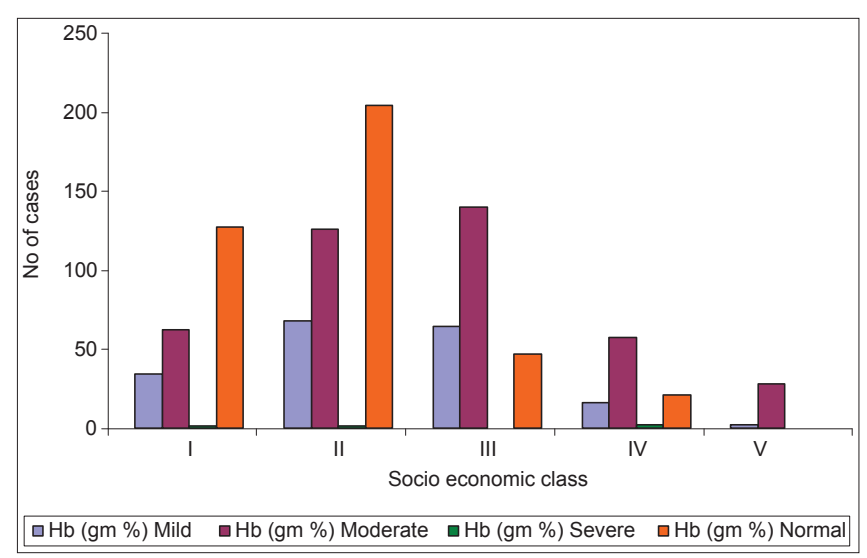

Figure 3: Association between socioecomomic class and Haemoglobin

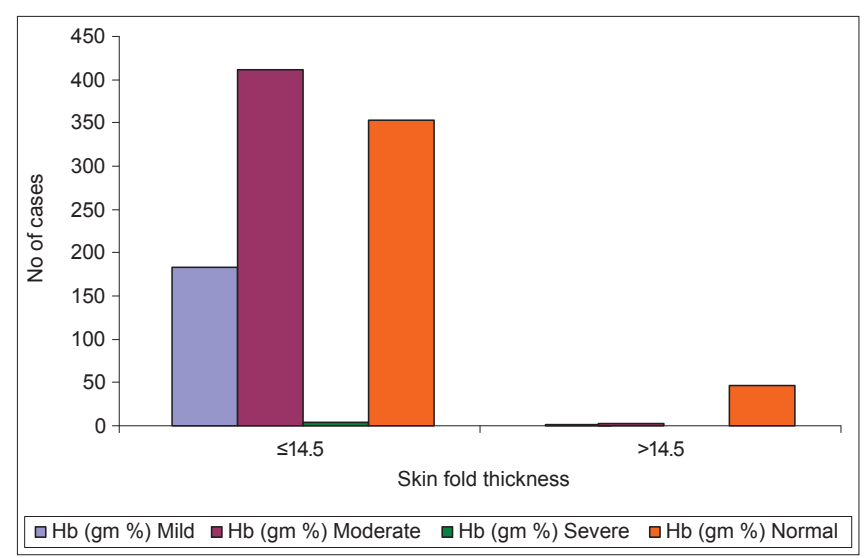

Figure 5: Association between skinfold thickness and Haemoglobin

In the present study, $70 \%$ of anemic subjects had low MCV with high RDW, suggestive of probably iron deficiency.

Body Mass Index (BMI) is the most practical and widely used tool to ascertain an individual's body fat mass. As per WHO BMI charts 2007, the subjects were classified, and it was observed that girls falling into category of $<3^{\text {rd }}$ to $15^{\text {th }}$ percentile had high prevalence of anemia. This is in consistent with the study conducted by Kannani and Poojara. ${ }^{[14]}$

Out of the observed 601 anemic subjects, 22 were found to have signs of other vitamin deficiencies such as Bitot spots in 8 and oral ulcers in 14 .

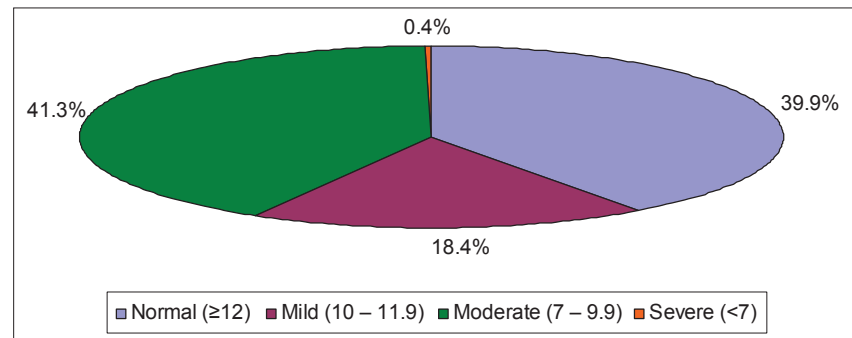

Figure 2: Haemoglobin wise distribution of cases

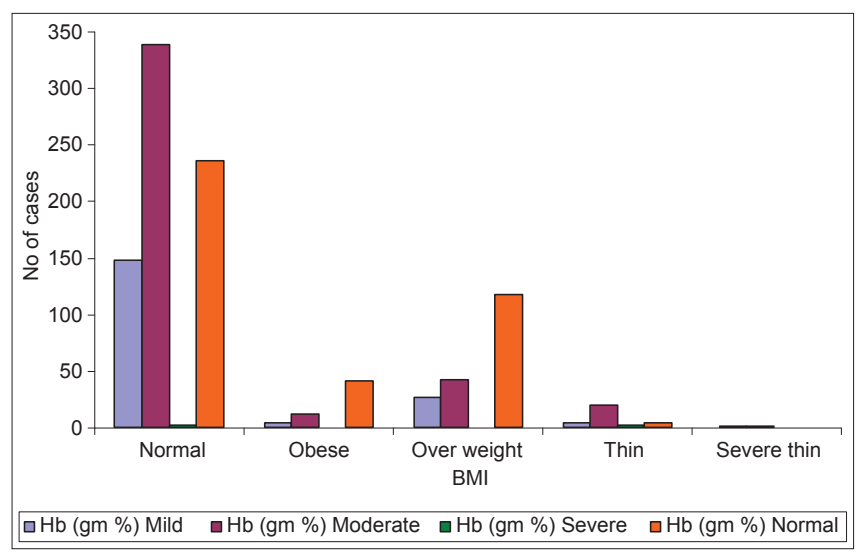

Figure 4: Association between BMI and Haemoglobin

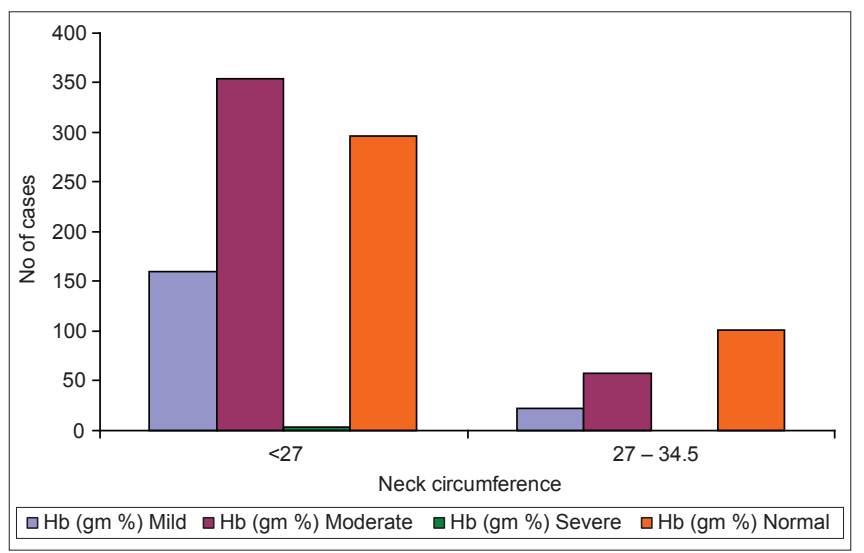

Figure 6: Association between neck circumference and Haemoglobin

\section{CONCLUSION}

- In the present study, $60 \%$ adolescent girls were found to be anemic

- The severity of anemia was found to be $18.4 \%$ as mild, $41.3 \%$ as moderate, and $0.4 \%$ as severe

- High prevalence of anemia was reported among girls belonging to class III, IV, and V as per modified Kuppuswami classification

- Thin and severely thin adolescents were found to at higher risk of anemia

- Four hundred and twenty out of $60 \%$ anemic adolescents had iron-deficiency anemia

- The present study delineates highly significant co-relation of BMI, SFT, NC with Hb levels. 


\section{ACKNOWLEDGMENTS}

1. Resident Dr. Priya Chauhan for collecting the data and updating proforma

2. Management and Dean of Dr.D. Y. Patil Medical College for allowing us to conduct this study

3. All our colleagues and laboratory staff for their assistance.

\section{REFERENCES}

1. Kumar N, Shekhar C, Kumar P, Kundu AS. Kuppuswamy's socioeconomic status scale-updating for 2007. Indian J Pediatr 2007;74:1131-2.

2. Guidelines for the use of iron supplements to prevent and treat iron deficiency anemia. Washington DC: International Anemia Consultative group; 1997.

3. WHO/UNICEF/UNU (2001) Iron deficiency anemia assessment; prevention and control. A guide for programme managers. Geneva: WHO/ UNICEF/UNU; 2001.

4. Available from: http://www.discovery.bits-pilani.ac.in. [Last accessed on 2013 July 12.

5. Gilleapie S. Major issues in the control of Iron deficiency Micronutrient Initiative. USA: UNICEF; 1998.

6. Kurtz KM, Welch CJ. The nutrition and lives of adolescent in developing countries: Findings from the nutrition of adolescent girls research programme. Washington, DC: International Centre for research on women; 1994.

7. Kaur S. Epidemiological correlates of nutritional anemia in adolescents girls of rural Wardha. Indian J Community Med 2006;31:4.
8. Rana T. Age at menarche - Nutritional status and other associated factors in Urban Hyderabad girls. Ph. d. thesis submitted to National INSTITUTE Of NUTRITION. Hyderabad: National INSTITUTE Of NUTRITION; 1983. p. 17.

9. Seshadri S. A database for iron deficiency anemia in India; prevalence, etiology, consequences and stratergies for control, Task force for micronutrients malnutrition control, Department of women and child development. New Delhi, India: Ministry of Human Resource Development; 1996.

10. Rawat CM, Garg SK, Singh VJ, Bhatnagar M, Chopra H, Bappai SK. Socio- demographic co- relates of anemia among adolescent girls in rural area of district Meerut (U.P). Indian J Community Med 2001;26:4.

11. Verma A, Rawal VS, Kedia G, Kumar D, Chauhan J. Factors influencing anemia among girls of school going age (6-18yrs) from slums of Ahmedabad City. Indian J Community Med 2004;24:1.

12. Gawarika R, Gawarika S, Mishra AK. Prevalence of anemia in adolescent girls belonging to different economic group. Indian J Community Med 2006;31:4.

13. Kapoor G, Aneja S. Nutritional disorders in adolescent girls. Indian Pediatr 1992;29:969-73.

14. Kanani SJ, Poojara RH. Supplementation with iron and folic acid enhances growth in adolescent Indian Girls J Nutr 2000;130:452-5S.

How to cite this article: Deshpande NS, Karva D, Agarkhedkar S, Deshpande S. Prevalence of anemia in adolescent girls and its co-relation with demographic factors. Int J Med Public Health 2013;3:235-9.

Source of Support: Nil, Conflict of Interest: None declared. 